\title{
BMJ Open The long-term effect of minimalist shoes on running performance and injury: design of a randomised controlled trial
}

\author{
Joel T Fuller, ${ }^{1}$ Dominic Thewlis, ${ }^{1}$ Margarita D Tsiros, ${ }^{1}$ Nicholas A T Brown, ${ }^{2}$ \\ Jonathan D Buckley ${ }^{1}$
}

To cite: Fuller JT, Thewlis D, Tsiros MD, et al. The longterm effect of minimalist shoes on running performance and injury: design of a randomised controlled trial. BMJ Open 2015;5:e008307. doi:10.1136/bmjopen-2015008307

- Prepublication history and additional material is available. To view please visit the journal (http://dx.doi.org/ 10.1136/bmjopen-2015008307).

Received 25 March 2015 Revised 24 July 2015 Accepted 30 July 2015

CrossMark

\section{${ }^{1}$ Alliance for Research in Exercise, Nutrition and Activity (ARENA), Sansom Institute for Health Research, University of South Australia, Adelaide, South Australia, Australia \\ ${ }^{2}$ Australian Institute of Sport, Bruce, Australian Capital Territory, Australia}

Correspondence to Joel T Fuller; joel.fuller@mymail. unisa.edu.au

\section{ABSTRACT \\ Introduction: The outcome of the effects of transitioning to minimalist running shoes is a topic of interest for runners and scientists. However, few studies have investigated the longer term effects of running in minimalist shoes. The purpose of this randomised controlled trial (RCT) is to investigate the effects of a 26 week transition to minimalist shoes on running performance and injury risk in trained runners unaccustomed to minimalist footwear.}

Methods and analysis: A randomised parallel intervention design will be used. Seventy-six trained male runners will be recruited. To be eligible, runners must be aged 18-40 years, run with a habitual rearfoot footfall pattern, train with conventional shoes and have no prior experience with minimalist shoes. Runners will complete a standardised transition to either minimalist or control shoes and undergo assessments at baseline, 6 and 26 weeks. $5 \mathrm{~km}$ time-trial performance (5TT), running economy, running biomechanics, triceps surae muscle strength and lower limb bone mineral density will be assessed at each time point. Pain and injury will be recorded weekly. Training will be standardised during the first 6 weeks. Primary statistical analysis will compare $5 \mathrm{TT}$ between shoe groups at the 6-week time point and injury incidence across the entire 26-week study period.

Ethics and dissemination: This RCT has been approved by the Human Research Ethics Committee of the University of South Australia. Participants will be required to provide their written informed consent prior to participation in the study. Study findings will be disseminated in the form of journal publications and conference presentations after completion of planned data analysis.

Trial registration number: This RCT has been registered with the Australian New Zealand Clinical Trials Registry (ACTRN12613000642785).

\section{INTRODUCTION}

The effects of running in minimalist shoes is a topic of interest for runners and for scientists. $^{1-7}$ Running in minimalist shoes can cause runners to run with a more

\section{Strengths and limitations of this study}

- This is the first study to investigate the effects of minimalist footwear over longer than a 3-month period and the first to include a measure of running performance.

- The standardised gradual transition to minimalist shoes from $0 \%$ to $100 \%$ of weekly running will inform runners, coaches and clinicians of the effect of these shoes across the entire transitional period.

- Limitations of the study are the inclusion of only male runners aged 18-40 years and the inclusion of only one minimalist shoe group.

plantar-flexed ankle at initial contact and adopt a forefoot footfall (FF) pattern, ${ }^{4} 67$ increase stride rate, ${ }^{17}$ reduce stride length, ${ }^{1}$ increase ankle plantar-flexor moments and decrease knee extensor moments, ${ }^{4}$ and improve running economy. ${ }^{2}$ Although athletes and coaches may be interested in the potential for minimalist shoes to improve running performance, there is also some evidence that minimalist shoes increase injury risk. ${ }^{5}$ However, few studies have included a prospective longitudinal follow-up of runners who changed from conventional to minimalist running shoes. ${ }^{5-11}$ At present, runners, coaches and clinicians attempting to make a more informed purchase or prescription of minimalist shoes are required to base their decision on predominantly acute studies with no longitudinal follow-up. ${ }^{1} 346$

The authors are not aware of any consensus on what should be an appropriate follow-up period to investigate the long-term effects of minimalist shoes. However, the few studies that have included a prospective longitudinal follow-up of runners who changed to minimalist shoes have used follow-up periods of only 4-12 weeks. ${ }^{5}$ 7-11 These 
studies found that, following the transition to minimalist shoes, runners improved running economy, ${ }^{7}$ reduced peak pressure under the heel ${ }^{9}$ and increased intrinsic foot muscle cross-sectional area, ${ }^{11}$ but experienced increased calf and shin pain, ${ }^{5} 8$ increased foot bone marrow oedema ${ }^{10}$ and a higher injury rate. ${ }^{5}$ It has been hypothesised that these effects of minimalist shoes result from runners adopting a FF pattern, which causes increased loading of musculoskeletal structures at the foot and ankle. ${ }^{11-13}$ If an appropriate, gradual transition to minimalist shoes can be made, it might be possible to derive beneficial training adaptations from this increased loading. ${ }^{71}$ However, if the increased loading is too rapid, pain and injury may result from the increased forces experienced by the ankle plantar-flexor muscles. ${ }^{4} 5$ Indeed, the only study to investigate the effect of transitioning to minimalist shoes on injury risk reported greater calf pain and a higher incidence of injury for two different minimalist shoes (20\% and $38 \%$ ) compared to conventional shoes $(13 \%) .^{5}$

Choice of running shoe is an important issue for distance runners who view minimalist shoes as a means for enhancing running performance but have concerns about the potential for minimalist shoes to cause injury. ${ }^{14}$ Avoiding injuries resulting from minimalist shoes is important for runners to minimise the economic burden associated with medical treatment and absenteeism from work, as well as to maximise the positive health effects of maintaining an active lifestyle. ${ }^{15}$ This issue is particularly relevant given the popularity of running, which is performed by $10 \%$ of the Australian population. ${ }^{16}$ In order to provide runners with sufficient instructions on how to implement minimalist shoes, further longitudinal studies investigating methods for transitioning to minimalist shoes over longer periods are needed. Current longitudinal studies involving minimalist shoes have either been limited to follow-up periods of 12 weeks or less, ${ }^{5-11}$ have only transitioned runners to using minimalist shoes for up to $60 \%$ of weekly running, ${ }^{5} 711$ have not used standardised methods for transitioning to minimalist shoes ${ }^{10}$ or have not investigated injury incidence ${ }^{7-11}$ and running performance, ${ }^{5} 8-11$ which are the two most significant outcomes for runners interested in minimalist shoes. ${ }^{14}$

\section{Primary objective}

The purpose of this randomised controlled trial (RCT) is to investigate the effects of a 26-week transition to minimalist shoes compared to conventional shoes on running performance and injury risk in trained runners unaccustomed to minimalist footwear. It is hypothesised that transitioning to minimalist shoes will have benefits for running performance but be associated with an increased risk of injury. The increased risk of injury is expected to result from the increased forces experienced by the ankle plantar-flexor muscles when running with a flatter foot position at initial contact in the minimalist shoe.
METHODS

This study protocol was developed according to the 2013 SPIRIT (Standard Protocol Items: Recommendations for Interventional Trials) statement. ${ }^{17}$ The study will use a two-arm RCT design with 6 and 26-week follow-up. Data collection will take place at the University of South Australia. The RCT has been registered with the Australian New Zealand Clinical Trials Registry. Trial registration data are shown in table 1 . Participants will be recruited from a sample of convenience on a volunteer basis. This study will be advertised at local universities, running clubs and running events. To assist with participant retention, participants will be provided with a $\$ 100$ shoe voucher if they successfully complete the 26-week study commitment. Participants who withdraw from the study due to injury will also receive the $\$ 100$ shoe voucher after independent assessment of the injury by a registered physiotherapist or medical practitioner not associated with the study.

\section{Study population}

Male runners will be recruited to avoid potential gender effects on running biomechanics. ${ }^{18}$ Only runners aged 18-40 years will be recruited because that is the most popular age group for participation in running in Australia. ${ }^{16}$ Participants will be required to run a minimum of $15 \mathrm{~km}$ per week $^{3}$ (this will minimise the risk of injuries resulting from a lack of familiarity with regular running ${ }^{19}$ ), be able to complete a $5 \mathrm{~km}$ treadmill time trial (5TT) in $\leq 23 \mathrm{~min}$ ( $95 \%$ of runners represented by the cohort of male endurance-trained runners in a study by Laursen et $a t^{20}$ would complete a 5 TT in less than $23 \mathrm{~min}$, ie, mean $5 \mathrm{TT}+2 \mathrm{SDs}$ was $23 \mathrm{~min}$ ), train with conventional running shoes, have no prior experience running in shoes with reduced cushioning, drop height and mass, run with a rearfoot footfall (RF) pattern at the time of enrolment in the study (typical of $89 \%$ of runners ${ }^{21}$ ) and have no current or recent (<3 months) musculoskeletal injury. Participants will be excluded if they have a history of invasive surgery to the back, pelvis or lower extremities in the previous year or if they use orthotics in their running shoes, because orthotics will not fit inside the minimalist shoe. Footfall pattern will be determined from overground running trials at a self-selected running speed in participants' own running shoes filmed at $200 \mathrm{~Hz}$ using a high-speed digital camera (Basler Pilot, Ahrensburg, Germany) to ensure only habitual RF runners are recruited.

\section{Sample size}

An a priori power calculation determined that 50 participants are required to detect a Cohen's d effect size of 0.3 for the primary outcome (5TT performance) at 6-week follow-up with $80 \%$ power and a 5\% significance level using analysis of covariance (ANCOVA). This calculation was performed using the formula described by Borm et $a t^{22}$ and South Australian $5 \mathrm{~km}$ road race results recorded in 2011 and 2012 (average race time $1139 \pm 140 \mathrm{~s}$ ). The 0.3 
Table 1 Trial registration data

\begin{tabular}{|c|c|}
\hline Data category & Information \\
\hline $\begin{array}{l}\text { Primary registry and trial } \\
\text { identifying number }\end{array}$ & https://www.anzctr.org.au ACTRN12613000642785 \\
\hline $\begin{array}{l}\text { Date of registration in primary } \\
\text { registry }\end{array}$ & 7th June, 2013 \\
\hline Secondary identifying numbers & None \\
\hline $\begin{array}{l}\text { Source of monetary or material } \\
\text { support }\end{array}$ & University of South Australia \\
\hline \multirow[t]{2}{*}{ Primary sponsor } & University of South Australia \\
\hline & Contact person: JDB (jon.buckley@unisa.edu.au) \\
\hline Secondary sponsor & None \\
\hline Contact for public queries & JTF (joel.fuller@mymail.unisa.edu.au) \\
\hline Contact for scientific queries & JTF (joel.fuller@mymail.unisa.edu.au) \\
\hline Public title & The effect of footwear on running performance and injury risk \\
\hline Scientific title & $\begin{array}{l}\text { In distance runners, do lightweight running shoes, compared to standard running shoes, } \\
\text { improve running performance and reduce risk of injury? }\end{array}$ \\
\hline Countries of recruitment & Australia \\
\hline Health problem studied & Running-related injury \\
\hline \multirow[t]{2}{*}{ Interventions } & Minimalist shoe (Asics Piranha SP4) \\
\hline & Conventional shoe (Asics Gel Cumulus-14, 15 or 16 ) \\
\hline \multirow[t]{2}{*}{$\begin{array}{l}\text { Key inclusion and exclusion } \\
\text { criteria }\end{array}$} & $\begin{array}{l}\text { Inclusion criteria: male, } 18-40 \text { years, running } \geq 15 \mathrm{~km} \text { per week, with habitual rearfoot } \\
\text { footfall and able to run a } 5 \mathrm{~km} \text { time trial in }<23 \mathrm{~min}\end{array}$ \\
\hline & $\begin{array}{l}\text { Exclusion criteria: prior experience with minimalist shoes, use of orthotics, having a current } \\
\text { or recent ( }<3 \text { months) musculoskeletal injury or history of recent }(<12 \text { months) invasive } \\
\text { surgery that affected running }\end{array}$ \\
\hline Study type & Randomised controlled trial \\
\hline Date of first enrolment & 24th June 2013 \\
\hline Target sample size & 76 \\
\hline Recruitment status & Closed: follow-up continuing \\
\hline Primary outcome & $5 \mathrm{~km}$ time trial performance (time point: 6 weeks) \\
\hline \multirow[t]{6}{*}{ Key secondary outcomes } & Injury incidence (time fame: 6 months) \\
\hline & $5 \mathrm{~km}$ time trial performance (time point: 6 months) \\
\hline & Running economy (time point: 6 weeks and 6 months) \\
\hline & Running biomechanics (time point: 6 weeks and 6 months) \\
\hline & Triceps surae strength (time point: 6 weeks and 6 months) \\
\hline & Bone mineral density (time point: 6 weeks and 6 months) \\
\hline
\end{tabular}

effect size was based on the 2.4-5.8\% improvement (mean improvement $3.6 \%$ ) in running economy observed for runners training with or experienced with minimalist shoes $^{6} 723$ and a corresponding $10.6 \mathrm{~m} / \mathrm{min}$ improvement in average race pace (44 $\mathrm{s}$ improvement in average race time) estimated by Burkett et al. ${ }^{24}$ To allow for a $20 \%$ drop out rate and a $25 \%$ rate of injury ${ }^{5}$ during the 6 -week follow-up period, it is expected that 76 participants will need to be recruited. An additional 12 participants will be recruited and will complete all outcome assessments on two separate occasions in their own shoes to determine test-retest reliability.

\section{Study protocol}

Participants will attend a familiarisation session in the week prior to their anticipated start date. During this session, information about previous injury and shoe use will be collected, participants will complete $30 \mathrm{~min}$ treadmill familiarisation, footfall pattern will be assessed and a 5TT will be completed. Randomisation to shoe group will be via a process of minimisation, ${ }^{25}$ using 5TT performance times obtained during familiarisation as the minimisation variable and a 1:1 allocation ratio. Allocation via minimisation offers the only acceptable alternative to simple and restricted randomisation, ${ }^{26}$ and is more effective at balancing the collective attributes of intervention groups in small samples than traditional methods of randomisation. ${ }^{27}$ Allocation of participants will be performed by one of the investigators (JDB) who will not be involved directly in data collection, but it will not be possible to blind outcome assessors to participant shoe condition during testing. Experimental testing sessions will be undertaken for each shoe condition at baseline, at 6-week follow-up and at 6-month follow-up. Outcomes assessed in order of assessment at each test session will be overground running kinematics and kinetics, treadmill running economy, bone mineral density (BMD), 5TT performance and muscle strength. A participant timeline is shown in figure 1. All testing sessions will be performed at the same time of the day. 


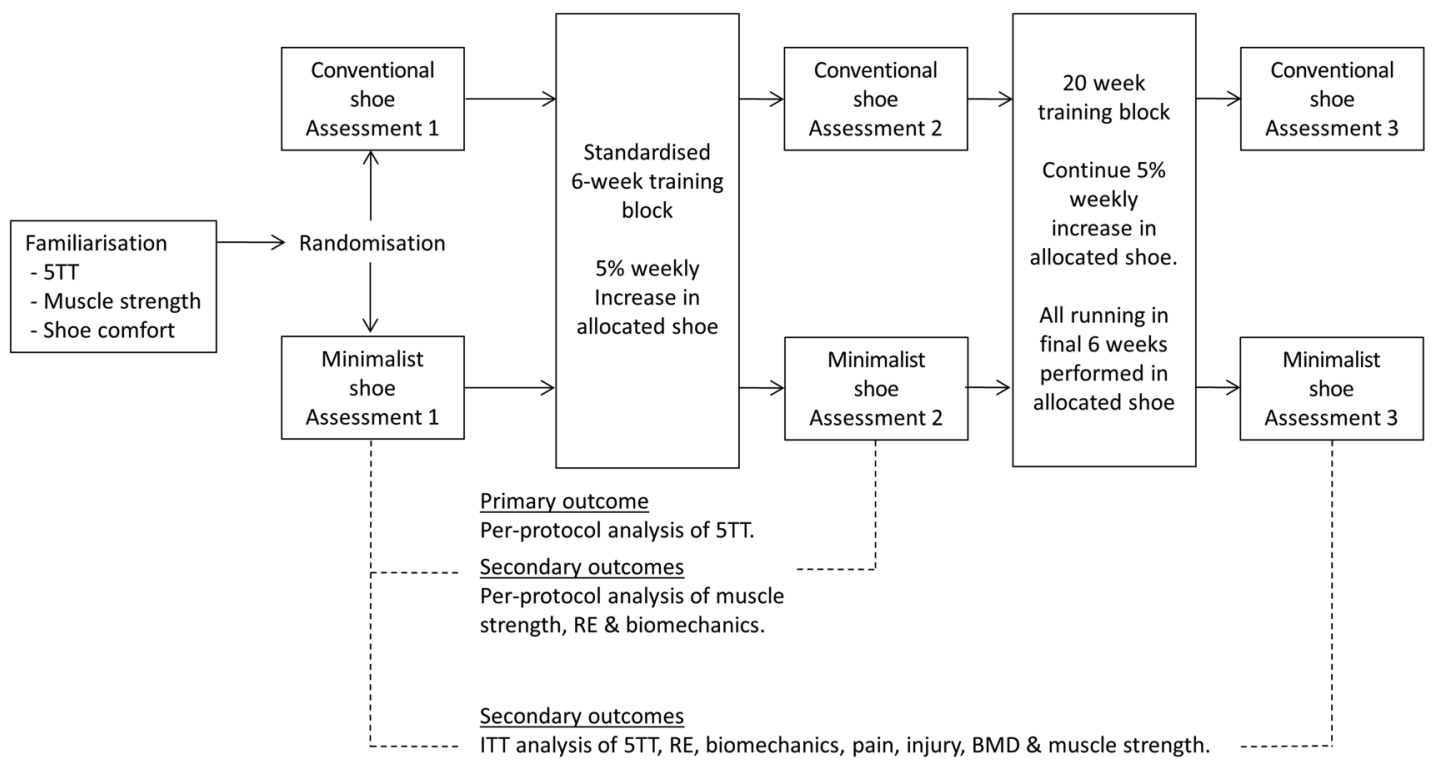

Figure 1 Participant timeline (BMD, bone mineral density; ITT, intention-to-treat; RE, running economy; $5 T$ T, 5 km treadmill time trial).

Participants will be required to not complete any training on the day of testing and remain fasted from food (water permitted ad libitum) in the $3 \mathrm{~h}$ prior to testing.

\section{Shoe conditions}

Participants allocated to the control condition will run in conventional running shoes (Asics Gel Cumulus-14, 15 or 16 ; mass $324 \mathrm{~g}$ /shoe; heel drop $9 \mathrm{~mm}$ ) and participants allocated to the minimalist shoe condition will run in lightweight racing flats (Asics Piranha SP4; mass $125 \mathrm{~g} /$ shoe; heel drop $5 \mathrm{~mm}$ ). Mass is reported for an average US size 9 (European size 42.5) shoe. Participants will be instructed to complete only $5 \%$ of running in their allocated shoes on each day that they run in the first week. This amount will then be increased by $5 \%$ each week until week 20 , when participants will complete $100 \%$ of running in the allocated shoes. From weeks 20-26, runners will perform all running in their allocated shoe condition.

To investigate how the runners perceive the comfort of the two shoe conditions, they will complete an assessment of shoe comfort for their respective shoe condition at the beginning of each testing session. Assessments of shoe comfort will be made using a $100 \mathrm{~mm}$ visual analogue scale (VAS) with anchors "not comfortable at all" on the left hand end and "most comfortable imaginable" on the right. ${ }^{28}$ Shoe comfort will be assessed before and following a 2 min submaximal run on a motorised treadmill at self-selected running speed (Model 645, Quinton Instrument Co, Washington, USA). Four familiarisation comfort assessments will be used to achieve stability of shoe comfort results. ${ }^{28}$

\section{Training programme}

Running training will be standardised during the first 6 weeks of the study so that relative training intensity and volume will be the same for all participants.
Training intensity will be prescribed relative to the peak heart rate $\left(\mathrm{HR}_{\text {peak }}\right)$ achieved during the 5TT. Participants will monitor training intensity throughout the training programme using a heart rate monitor (Polar F1 heart rate monitor, Polar Electro Oy, Kempele, Finland). The training programme is adapted from a 6-week training programme used by Billat et $a l^{29}$ that was shown to increase $\mathrm{VO}_{2 \max }$ by $3.6 \%$ in trained runners. The training programme is described in table 2. During weeks 7-26, training will not be standardised and participants will complete their usual training to evaluate the effects of the shoe under non-controlled conditions so as to provide ecologically valid outcome data, but the transition to the allocated shoe will continue to be increased by $5 \%$ per week during this period until participants are completing $100 \%$ of training in the allocated shoe. Adherence to training and shoe allocation will be monitored using a participant training diary. Participants reporting an injury during the study period will be advised to stop the gradual increase in allocated shoe use until they have recovered. Injured participants will be invited to have their injury assessed and treated at the University of South Australia physiotherapy clinic. No study investigators will be involved in the assessment or treatment of injured participants.

\section{Running kinematics and kinetics}

Running kinematics and kinetics will be assessed during overground running trials performed at $18 \mathrm{~km} / \mathrm{h} \pm 10 \%$ over a $40 \mathrm{~m}$ runway. Consistency of running speed will be monitored using photoelectric sensors (SpeedLight V2, Swift Performance Equipment, Queensland, Australia). Marker trajectories will be measured using a 12 camera VICON MX F20 system (Vicon, Oxford, UK) sampling at $300 \mathrm{~Hz}$. Ground reaction force will be measured using four force platforms aligned in series and sampling at $1200 \mathrm{~Hz}$. Each participant will be required 
Table 2 Six-week standardised running training programme

\begin{tabular}{|c|c|c|c|c|c|c|c|c|}
\hline \multirow[b]{2}{*}{ Week } & \multirow[b]{2}{*}{ Method } & \multicolumn{7}{|c|}{ Training session duration (min) } \\
\hline & & Day 1 & Day 2 & Day 3 & Day 4 & Day 5 & Day 6 & Day 7 \\
\hline \multirow[t]{2}{*}{1} & LSD & - & 50 & - & - & 50 & - & - \\
\hline & HIIT & $3 \times 10$ & - & - & $2 \times 15$ & - & - & - \\
\hline \multirow[t]{2}{*}{2} & LSD & - & 50 & - & - & 50 & - & - \\
\hline & HIIT & $3 \times 12$ & - & - & $2 \times 18$ & - & - & - \\
\hline \multirow[t]{2}{*}{3} & LSD & - & 50 & - & - & 50 & - & - \\
\hline & HIIT & $3 \times 14$ & - & - & $2 \times 21$ & - & - & - \\
\hline \multirow[t]{2}{*}{4} & LSD & - & 50 & - & - & 50 & - & - \\
\hline & HIIT & $3 \times 16$ & - & - & $2 \times 24$ & - & - & - \\
\hline \multirow[t]{2}{*}{5} & LSD & - & 50 & - & - & 50 & - & - \\
\hline & HIIT & $3 \times 18$ & - & - & $2 \times 27$ & - & - & - \\
\hline \multirow[t]{2}{*}{6} & LSD & - & - & 40 & - & 40 & - & - \\
\hline & HIIT & $3 \times 20$ & - & - & - & - & - & - \\
\hline
\end{tabular}

LSD, long slow distance running at $65-80 \%$ peak heart rate $\left(\mathrm{HR}_{\text {peak }}\right) ; \mathrm{HIIT}$, high-intensity interval training running at $85-90 \% \mathrm{HR}$ peak. Intervals separated by 5 min of walking.

to complete five successful trials. A trial will be considered successful if the full plantar surface of the foot contacts the force platform in between the pylons of the force platform at the prescribed running speed without obvious modification of gait. Participants will not be provided with any instructions in regard to contacting the force platform. Instead, the runway starting point will be adjusted as needed to facilitate a successful trial.

A full body marker set-up will be used and will include the feet, shanks, thighs, pelvis, trunk (including head), upper arms and lower arms (including hands). Spherical retroreflective calibration markers will be used to define the position and orientation in space (POSE) of each segment, and will be placed over the first and fifth metatarsal head, lateral and medial malleolus, lateral and medial femoral epicondyle, greater trochanter, anterior superior iliac spine, posterior superior iliac spine C7 spinous process, acromioclavicular joint, lateral and medial humeral epicondyle, and radial and ulnar styloid process. Tracking markers will be used to track the POSE of each segment. A minimum of three noncollinear markers will be used to track each segment in six degrees of freedom. This marker set-up will be used to investigate footfall pattern as well as kinematics and kinetics at the knee and ankle.

\section{Running economy}

Participants will complete a 5 min warm-up on the treadmill at $8 \mathrm{~km} / \mathrm{h}$. Running economy will then be assessed during three $6 \mathrm{~min}$ submaximal runs on the treadmill at 11,13 and $15 \mathrm{~km} / \mathrm{h}$ in a fixed order. Running economy will be assessed by indirect calorimetry (True One, ParvoMedics, Utah, USA) and expressed as the rate of energy expenditure (REE; $\mathrm{kJ} / \mathrm{min}$ ) during the final $60 \mathrm{~s}$ of each 6 min run. Participant data will be excluded if the respiratory exchange ratio (RER) increases above 1 during the final $60 \mathrm{~s}$ or a steady state of oxygen consumption is not achieved.

\section{Spatiotemporal parameters}

Force-sensitive resistors (FSR) will be placed underneath the heel and forefoot regions of each shoe, and used to assess footfall pattern, stride rate and stride length during each 6 min run. Foot contacts will be recorded wirelessly at $2000 \mathrm{~Hz}$ using a Delsys Trigno system (DelSys Inc, Natick, USA). Runners will be classified with a RF pattern if initial contact is made with the FSR positioned at the heel or a FF pattern if initial contact is made with the FSR positioned at the forefoot. Footfall pattern will be assessed throughout each 6 min run and classification will be based on the mode (ie, whichever pattern is most frequent) during the final $60 \mathrm{~s}$. Stride rate (strides per minute) will be considered the number of right foot contacts recorded during the final $60 \mathrm{~s}$ of each $6 \mathrm{~min}$ run. Average stride length will be calculated using the following equation:

$$
\mathrm{SL}=\mathrm{ST} \times \mathrm{V}
$$

SL is the stride length, ST the time taken for each stride (right foot contact to right foot contact) and $\mathrm{V}$ the treadmill speed ${ }^{30}$ from the known distance covered during the $60 \mathrm{~s}$ because speed will remain fixed. Footfall pattern, stride length and stride rate computations will be performed using a custom written code in MATLAB (R2013a, MathWorks, Massachusetts, USA).

\section{$5 \mathrm{~km}$ treadmill time trial}

Running endurance performance will be assessed using a 5TT on a motorised treadmill set at $0 \%$ grade. Participants will be instructed to complete the 5TT in the fastest possible time and will be free to adjust the treadmill speed throughout the test. Starting speed will remain constant across testing sessions and will be selected by participants prior to completion of their baseline 5TT. Participants will be blinded to treadmill speed and time throughout performance of the 5TT. 
Peak oxygen consumption during the 5TT will be assessed by indirect calorimetry.

\section{Bone mineral density}

BMD of the right proximal tibia, calcaneus and metatarsals will be measured by dual X-ray absorptiometry (Lunar Prodigy, General Electric Corporation, Madison, USA) using two separate scans. These peripheral sites were chosen for the assessment of BMD because it was hypothesised that they would be most likely to change in response to the potential alterations in running biomechanics that can result from running in minimalist shoes. ${ }^{46}$ For assessment of the tibia and calcaneus, participants will be positioned in a side lying position. ${ }^{31}$ For assessment of the metatarsals, participants will be positioned in an upright, seated position with the foot in the plantar position. ${ }^{32}$ For each participant, goniometer measurements for the knee and ankle in the sagittal plane will be recorded and standardised between scans to help reproduce the same scan position.

\section{Muscle strength}

Muscle strength assessment of the triceps surae muscle group will be performed on an isokinetic dynamometer (Biodex System 4, Biodex Medical Systems, New York, USA) with participants in a reclined seated position with knees positioned in $20-30^{\circ}$ flexion. Peak isometric torque will be measured with the ankle positioned in the anatomical neutral position and defined as the peak torque achieved during the better of two $5 \mathrm{~s}$ efforts. Peak concentric torque (PCT) and peak eccentric torque (PET) will be measured at an angular velocity of $30^{\circ} / \mathrm{s}$. Two sets of three repetitions will be performed for both PCT and PET measurements, with the peak torque achieved across repetitions considered the participant's PCT and PET. Torque data will be sampled at $1000 \mathrm{~Hz}$ using a PowerLab data acquisition system (PowerLab 16/30, ADInstruments, Bella Vista, Australia).

\section{Pain and injury monitoring}

Pain will be assessed using a study diary for seven regions of interest using a $100 \mathrm{~mm}$ VAS with anchor points consisting of 'no pain' on the left hand end and 'worst pain' on the right hand end. Regions of interest will include the foot, ankle, calf, shin, knee, thigh and lumbopelvic area. At the end of each week, participants will be asked to record the worst running-related pain that they experienced during the previous 7 days for each of the 7 regions of interest. A difference in VAS score of $10 \mathrm{~mm}$ will be considered clinically significant. ${ }^{33}$ An injury will be considered to be any musculoskeletal problem that is attributed by the participant to running. The problem will need to be severe enough to cause a reduction in weekly running distance, a visit to a health professional or the use of medication. ${ }^{34} 35$ Injuries attributed to an accident will not be considered. Injury events will be reported to the University of South Australia
Human Research Ethics Committee who will independently monitor the safety of the study interventions.

\section{Data management}

Outcome data will be entered electronically and stored in a password protected folder on the University of South Australia network server at the time of collection. Only study investigators will have access to the data and investigators will meet weekly to monitor progress of data collection. All outcome data will be de-identified using a participant identification numbering system.

\section{Statistical methods}

Per-protocol analysis will be used to assess the effect of shoe group at the end of the 6-week standardised training programme. Participants unable to complete a follow-up assessment at the end of the 6-week training programme due to injury will not be included in the per-protocol analysis. An ANCOVA will be used to compare 5TT performance, running kinematics, and kinetics and muscle strength between shoe groups after adjusting for baseline time. Running economy and spatiotemporal parameters will be analysed using a linear fixed-effects model with independent variables shoe, speed, time and shoe*speed*time interaction. Shoe mass will be included as a covariate in the statistical model for running economy. Cases with missing running economy data (ie, RER $>1.00$ ) will be included in the model.

Intention-to-treat analysis will be used to assess the effect of shoe group at the end of the 26-week study period. 5TT performance, running economy, muscle strength, BMD, spatiotemporal parameters, and running kinematics and kinetics will be compared between shoe groups using a linear fixed-effects model with independent variables shoe, time and shoe*time interaction. The independent variables speed and shoe*speed*time will be included in the statistical models for running economy and spatiotemporal parameters. Shoe mass will be included as a covariate in the statistical model for running economy. Cases with missing running economy data (ie, RER >1.00) will be included in the model. Injury rate will be analysed by $\log$ binomial generalised linear model with independent variable group. Sensitivity analysis will be undertaken to determine whether the effect of shoes is influenced by adherence to training programme and training volume, by including adherence and training volume as covariates. All statistical analysis will be performed in SPSS (V.22, IBM, New York, USA). Statistical significance will be assumed for $\mathrm{p}<0.05$.

\section{ETHICAL CONSIDERATIONS}

This protocol has received ethical approval from the Human Research Ethics Committee of the University of South Australia. The Ethics Committee will be notified of any planned amendments to the original protocol. 
Amendments will not be made without the prior approval of the Ethics Committee and all members of the investigatory team.

JTF will manage all expressions of interests and will provide all potential participants with a study information sheet. At the beginning of the initial familiarisation session, participants will be briefed on all aspects of the study and provided with an opportunity to have any questions answered. Eligible participants will be required to provide their written informed consent prior to participation in the study (see online supplementary material).

\section{DISSEMINATION OF FINDINGS}

Study results will be released to participants in a de-identified format. Participants will be provided with a separate copy of their personal results. Study findings will be released to the public in the form of journal publications and conference presentations.

\section{DISCUSSION}

Prospective longitudinal follow-up of runners who change to minimalist shoes is important to inform runners, coaches and clinicians about their safety and efficacy. Although short-term studies are informative for describing any immediate effects of minimalist shoes on running parameters, they are not useful for informing evidence-based prescription for longer term use. ${ }^{36}$ We propose to use an RCT to provide high-quality evidence regarding the efficacy of transitioning from conventional shoes to minimalist shoes.

There is still no consensus definition for minimalist shoes. ${ }^{5}$ Instead, footwear is considered to be minimalist if shoe mass, heel drop and cushioning are reduced compared with conventional running shoes. ${ }^{15}$ Choice of minimalist shoe is an important consideration for studies investigating the effects of these shoes. In this RCT, a racing flat will be used as the minimalist shoe condition. Racing flats differ from conventional running shoes by having reduced shoe mass, heel drop and cushioning, and, as such, can be categorised as a form of minimalist shoe. ${ }^{1}$ Additionally, racing flats have been used by runners and coaches in competition for many years, ${ }^{1} 37$ and can be considered representative of the footwear condition that runners used prior to the introduction of the modern conventional running shoe, which has increased shoe mass, heel drop and cushioning. ${ }^{14}$ It has been suggested that the introduction of the modern conventional running shoe may have caused changes to the natural human running gait and resulted in an increased injury rate. ${ }^{38}$ To adequately test this hypothesis, conventional running shoes should be compared with racing flats, which were the predominant running shoe available to runners prior to the introduction of shoe cushioning and heel raise.

It has been proposed that carefully transitioning from conventional to minimalist running shoes can avoid injuries attributed to sudden changes in footwear. ${ }^{5}$ 7-11 Several methods have been suggested for making this transition $^{5}{ }^{7-11}$ (table 3), but currently, there is insufficient evidence available on which to base informed recommendations. The percentage of running performed in minimalist shoes during the first week of transition has ranged from 3-33\% and has then been progressed each week by small amounts (table 3)..$^{5-11}$ Across studies, runners progressed the volume of minimalist shoe running by $3-20 \%$ each week (table 3). ${ }^{5} 78{ }^{2} 10 \quad 11$ This heterogeneity across

Table 3 Methods of transitioning from conventional to minimalist footwear used in the literature

\begin{tabular}{|c|c|c|c|c|}
\hline Author & Date & Minimalist shoe & Week 1* & Method for transitioning to minimalist footwear* \\
\hline Giandolini et aß & 2013 & Salomon Sense S-Lab & $33 \%$ & $\begin{array}{l}\text { Increase by } 3-17 \% \text { each week until reaching } 100 \% \text { in } \\
\text { week } 4 \\
100 \% \text { of running performed in minimalist shoes from week } \\
5-12\end{array}$ \\
\hline Ridge et $a l^{10}$ & 2013 & Vibram FiveFingers & $3-13 \%$ & $\begin{array}{l}\text { Increase by } 3-13 \% \text { each week until week } 3 \\
\text { Participants made further increases as they felt } \\
\text { comfortable during weeks } 4-10\end{array}$ \\
\hline Ryan et $a{ }^{\Gamma}$ & 2013 & $\begin{array}{l}\text { Vibram FiveFingers } \\
\text { Nike Free V.5.0 }\end{array}$ & $19 \%$ & $\begin{array}{l}\text { Gradual increases were made from week } 1-12 \\
58 \% \text { of running performed in minimalist shoes during week } \\
12\end{array}$ \\
\hline Miller et al ${ }^{11}$ & 2014 & $\begin{array}{l}\text { New Balance Road } \\
\text { Minimus } \\
\text { Merrel Pace/Trail } \\
\text { Glove }\end{array}$ & $7 \%$ & $\begin{array}{l}\text { Increase by }<10 \% \text { each week from week } 1-12 \\
\text { Up to } 43 \% \text { of running performed in minimalist shoes during } \\
\text { final weeks }\end{array}$ \\
\hline $\begin{array}{l}\text { Warne and } \\
\text { Warrington }\end{array}$ & 2014 & Vibram FiveFingers & $10 \%$ & $\begin{array}{l}\text { Gradual increases were made from week } 1-4 \\
25 \% \text { of running performed in minimalist shoes during } \\
\text { week } 4\end{array}$ \\
\hline Moore et aP & 2015 & Vibram FiveFingers & $\begin{array}{l}3-10 \\
\text { miles }\end{array}$ & $\begin{array}{l}\text { Increase by no more than } 20 \% \text { each week } \\
\text { No running performed in conventional shoes throughout } \\
\text { the transition }\end{array}$ \\
\hline
\end{tabular}


studies suggests that it is currently unclear what is an appropriate rate of progression for transition to running in minimalist shoes. In the present study, runners will use a 5\% per week progression in the amount of time spent running in minimalist shoes until they reach $100 \%$ (by week 20). They will then continue to run $100 \%$ in minimalist shoes during the final 6 weeks of the study. Onset of injury and weekly pain scores will be used to determine if there is a threshold amount of running in minimalist shoes that is associated with an increased risk of injury in runners transitioning from conventional shoes. This will provide important information to runners, coaches and clinicians who are planning a transition to minimalist shoes about optimal transition rates to reduce the risk of injury.

To maximise recruitment and to allow for a longer follow-up period, as well as in order to achieve ecological validity, this RCT will use a period of nonstandardised training between 6 and 26 weeks of follow-up, during which participants will perform their own usual training programme. The outcomes at week 26 are likely to be affected by the different training regimes followed by participants during this nonstandardised phase between weeks 6 and 26, but any heterogeneity of usual training regimes should be balanced across groups through the randomisation process.

The efficacy of minimalist shoes for improving performance and their safety in terms of injury risk is thought to be influenced by the running kinematics that are adopted when running in this type of footwear. ${ }^{5} 7$ Running in minimalist shoes has previously been shown to reduce stride length ${ }^{1}$ and the amount of ankle dorsiflexion at initial ground contact, ${ }^{6}$ with the latter promoting a FF pattern. ${ }^{7}$ Changing to a FF pattern increases ankle joint contact forces and plantar-flexor muscle forces. ${ }^{13}$ Increased involvement of the ankle plantarflexor muscle could result in greater elastic energy storage and recovery in the Achilles' tendon, which may contribute to improved running economy and performance. ${ }^{49}$ We hypothesise that the increased loading on these structures may contribute to greater adaptation with resultant greater increases in performance in response to training. ${ }^{7}$ However, these unaccustomed high forces could also increase risk of injury until sufficient adaptation has occurred in muscular and articular tissue. ${ }^{5} 10^{11} 13$ Previous research has observed changes in muscle and bone tissue when transitioning to minimalist shoes over $10-12$ weeks, ${ }^{10}{ }^{11}$ and the 26 week transition used in the present study will add to this knowledge. Additionally, examining the effects of minimalist shoes on running biomechanics, muscle strength, BMD and running economy in the present study will allow for investigation of the factors underlying any effects on performance and/or injury to be explored.

\section{CONCLUSION}

In conclusion, this RCT will provide high-quality evidence regarding the effect on running performance of transitioning from conventional to minimalist shoes, which is currently lacking in the field of running footwear research. Additionally, observation of injury rates during this transition will be used to inform the design of larger studies investigating the effect of minimalist shoes on injury risk. The 26-week longitudinal follow-up period used in this study will be the longest prospective follow-up of runners changing to minimalist shoes that has been reported in the literature to date. Assessments of running biomechanics, muscle strength and BMD will allow this RCT to explore the mechanisms underlying any effects of minimalist shoes on running performance and/or injury.

Acknowledgements The authors would like to thank ASICS 0ceania (ASICS Oceania Pty Ltd, Eastern Creek, NSW, Australia) for donating 20 pairs of Asics Gel Cumulus-16 running shoes to support this research. No other sources of industry support have been provided to support the completion of this RCT. Purchase of footwear will be arranged through a local running shoe store (Jogger's World, Adelaide SA, Australia) using funds obtained from a University of South Australia Vice Chancellor and President's Scholarship $(\$ 10,000)$ awarded to JTF.

Contributors JTF, DT, MDT, NATB and JDB conceived the study, participated in its design and helped to draft this manuscript. JTF will be responsible for data collection and statistical analysis.

Funding JTF is the recipient of an Australian Postgraduate Award from the Australian Commonwealth Government.

Competing interests DT has been a recipient of funding from ASICS Oceania (ASICS Oceania Pty Ltd, Eastern Creek, NSW, Australia) to undertake separate research.

Ethics approval Human Research Ethics Committee of the University of South Australia.

Provenance and peer review Not commissioned; externally peer reviewed.

Open Access This is an Open Access article distributed in accordance with the Creative Commons Attribution Non Commercial (CC BY-NC 4.0) license, which permits others to distribute, remix, adapt, build upon this work noncommercially, and license their derivative works on different terms, provided the original work is properly cited and the use is non-commercial. See: http:// creativecommons.org/licenses/by-nc/4.0/

\section{REFERENCES}

1. Bonacci J, Saunders PU, Hicks A, et al. Running in a minimalist and lightweight shoe is not the same as running barefoot: a biomechanical study. Br J Sports Med 2013;47:387-92.

2. Fuller JT, Bellenger CR, Thewlis D, et al. The effect of footwear on running performance and running economy in distance runners: a systematic review. Sports Med 2015;45:411-22.

3. Hamill J, Russell EM, Gruber AH, et al. Impact characteristics in shod and barefoot running. Footwear Sci 2011;3:33-40.

4. Paquette MR, Zhang S, Baumgartner LD. Acute effects of barefoot minimal shoes and running shoes on lower limb mechanics in rear and forefoot strike runners. Footwear Sci 2013;5:9-18.

5. Ryan M, Elashi M, Newsham-West R, et al. Examining injury risk and pain perception in runners using minimalist footwear. $\mathrm{Br} J$ Sports Med 2013;48:1257-62.

6. Squadrone R, Gallozzi C. Biomechanical and physiological comparison of barefoot and two shod conditions in experienced barefoot runners. J Sports Med Phys Fitness 2009;49:6-13.

7. Warne J, Warrington GD. Four-week habituation to simulated barefoot running improves running economy when compared with shod running. Scand J Med Sci Sports 2014;24:563-8.

8. Giandolini M, Horvais N, Farges $\mathrm{Y}$, et al. Impact reduction through long-term intervention in recreational runners: midfoot strike pattern versus low-drop/low-heel height footwear. Euro J Appl Physiol 2013;113:2077-90. 
9. Moore IS, Pitt W, Nunns M, et al. Effects of a seven-week minimalist footwear transition programme on footstrike modality, pressure variables and loading rates. Footwear Sci 2015;7:17-29.

10. Ridge ST, Johnson AW, Mitchell UH, et al. Foot bone marrow edema after a 10-wk transition to minimalist running shoes. Med Sci Sports Exerc 2013;45:1363-8.

11. Miller EE, Whitcome KK, Lieberman DE, et al. The effect of minimal shoes on arch structure and intrinsic foot muscle strength. J Sport Health Sci 2014;3:74-85.

12. Hamill J, Gruber AH, Derrick TR. Lower extremity joint stiffness characteristics during running with different footfall patterns. Euro $J$ Sport Sci 2014;14:130-6.

13. Rooney BD, Derrick TR. Joint contact loading in forefoot and rearfoot strike patterns during running. J Biomech 2013;46: 2201-6.

14. Rothschild CE. Primitive running: a survey analysis of runners' interest, participation, and implementation. J Strength Cond Res 2012;26:2021-6.

15. Samitz G, Egger M, Zwahlen M. Domains of physical activity and all-cause mortality: systematic review and dos-response meta-analysis of cohort studies. Int J Epidemiol 2011;40:1382-400.

16. Australian Sports Commission. Participation in exercise, recreation and sport: annual report. Canberra: Australian Government, 2010. Viewed 12th June 2015. http://www.ausport.gov.au/_data/assets/ pdf_file/0018/436122/ERASS_Report_2010.PDF

17. Chan AW, Tetzlaff JM, Altman DG, et al. SPIRIT 2013 statement: defining standard protocol items for clinical trials. Ann Intern Med 2013;158:200-7.

18. Ferber R, Davis IM, Williams DS. Gender differences in lower extremity mechanics during running. Clin Biomech 2003;18:350-7.

19. Buist I, Bredeweg SW, Bessem B, et al. Incidence and risk factors of running-related injuries during preparation for a 4-mile recreational running event. Br J Sports Med 2010;44:598-604.

20. Laursen PB, Francis GT, Abbiss CR, et al. Reliability of time-to-exhaustion versus time-trial running tests in runners. Med Sci Sports Exerc 2007;39:1374-9.

21. Larson $\mathrm{P}$, Higgins $\mathrm{E}$, Kaminski J, et al. Foot strike patterns of recreational and sub-elite runners in a long-distance road race. J Sports Sci 2011;29:1665-73.

22. Borm GF, Fransen J, Lemmens WAJG. A simple sample size formula for analysis of covariance in randomized clinical trials. J Clin Epidemiol 2007:60:1234-8

23. Perl DP, Daoud AI, Lieberman DE. Effects of footwear and strike type on running economy. Med Sci Sports Exerc 2012;44:1335-43.
24. Burkett LN, Kohrt WM, Buchbinder R. Effects of shoes and foot orthotics on VO2 and selected frontal plane kinematics. Med Sci Sports Exerc 1985;17:158-63.

25. Altman DG, Bland JM. Treatment allocation by minimisation. BMJ 2005;330:843.

26. Moher D, Hopewell S, Schulz KF, et al. CONSORT 2010 explanation and elaboration: updated guidelines for reporting paralle group randomised trials. J Clin Epidemiol 2010;63:1-37.

27. Scott NW, McPherson GC, Ramsay CR, et al. The method of minimization for allocation to clinical trials: a review. Control Clin Trials 2002;23:662-74.

28. Mills K, Blanch P, Vicenzino B. Identifying clinically meaningful tools for measuring comfort perception of footwear. Med Sci Sports Exerc 2010;42:1966-71

29. Billat $V$, Sirvent $P$, Lepretre PM, et al. Training effect on performance, substrate balance and blood lactate concentration at maximal lactate steady state in master endurance-runners. Euro $J$ Physiol 2004;447:875-83.

30. Cavanagh PR, Williams KR. The effect of stride length variation on oxygen uptake during distance running. Med Sci Sports Exerc 1982;14:30-5.

31. Sievänen H, Oja P, Vuori I. Precision of dual-energy X-ray absorptiometry in determining bone mineral density and content of various skeletal sites. J Nucl Med 1992;33:1137-42.

32. Sindel M, Keleş-Coşkun N, Melikoğlu MA, et al. Bone mineral density of the metatarsal bones and the first ray in male sportsmen. Int J Exp Clin Anat 2010;4:39-44.

33. Kelly $A$. The minimum clinically significant difference in visual analogue scale pain score does not differ with severity of pain Emerg Med J 2001;18:205-7.

34. Macera CA, Pate RR, Powell KE, et al. Predicting lower-extremity injuries among habitual runners. Arch Intern Med 1989;149:2565-8.

35. Walter SD, Hart LE, McIntosh JM, et al. The Ontario cohort study of running-related injuries. Arch Intern Med 1989;149:2561-4.

36. Richards CE, Magin PJ, Callister R. Is your prescription of distance running shoes evidence-based? Br J Sports Med 2009:43:159-62.

37. Hamill J, Freedson PS, Boda W, et al. Effects of shoe type on cardiorespiratory responses and rearfoot motion during treadmill running. Med Sci Sports Exerc 1988;20:515-21.

38. Lieberman DE. What we can learn about running from barefoot running: an evolutionary medical perspective. Exerc Sport Sci Rev 2012:40:63-72.

39. Saunders PU, Pyne DB, Telford RD, et al. Factors affecting running economy in trained distance runners. Sports Med 2004;34:465-85. 